\title{
Hardware Implementation of Hybrid Wind-Solar Energy System for Pumping Water Based on Artificial Neural Network Controller
}

\author{
Ons ZARRAD ${ }^{1 *}$, Mohamed Ali HAJJAJI ${ }^{2,3}$, Mohamed Nejib MANSOURI ${ }^{1}$ \\ ${ }^{1}$ Unit of Industrial Systems Study and Renewable Energy, National Engineering School, \\ University of Monastir, Monastir 5000, Tunisia \\ Zarrad_ons@yahoo.fr(*Corresponding author), mansouri.nejib@hotmail.fr \\ ${ }^{2}$ Laboratory of Electronic and Microelectronic, University of Monastir, Monastir 5000, Tunisia \\ ${ }^{3}$ Higher Institute of Applied Sciences and Technology of Kasserine, University of Kairouan, \\ Kairouan 3100, Tunisia \\ daly_fsm@yahoo.fr
}

\begin{abstract}
Solar energy and wind energy are being used more and more as a renewable source by various countries for different purposes. These energies offer many advantages and have a unique limitation due to the instability of energy. The aim of this paper is to command and synchronize the power flow of one hybrid system using two sources of energy (solar and wind). The first contribution of the present work is represented by the utilization of an Artificial Neural Network controller to command the maximum power point at fixed atmospheric conditions. The second contribution is represented by the optimization of the system respecting real-time constraints in order to increase the generating system performance. For this, the simulation and hardware implementation of the proposed algorithm are accomplished using MATLAB/SIMULINK and a Xilinx System Generator. The simulation results confirm that the considered system presents acceptable execution real time performance and precision. The proposed designed model and its control strategy give the opportunity to optimize the hybrid power system performance, which is utilized in rural pumping applications.
\end{abstract}

Keywords: Wind turbine system, PV, MPPT, Artificial Neural Network controller, FPGA, Pumping water.

\section{Introduction}

Water is absolutely necessary for life and the need of water increases more and more with the rise in world population (Sharma \& Pritamdas, 2016). Pumping water is very important to clean water for drinking and irrigate agricultural fields. The expensive installation of the grid to pump water in rural villages is also considered a big problem in many developing countries. Furthermore, transporting fuel, needed for water pumping (Gupta, Vaishnav \& Garg, 2015), in rural villages can be difficult, unsafe and with limited quantity. The most attractive energy used in rural areas to pump water is the renewable energy, such as Photovoltaic (PV) panels and wind machines. Thanks to the importance of these renewable energies, the combination of these two intermittent sources can guarantee the stability and continuity of the energy production. Regardless of day or night, a wind energy resource is always there (Belaji Damodhar \& Senthil Kumar, 2016). The solar energy resource is present only in daytime, but because solar energy is continuous and stable, it can make up for the intermittent characteristics and the discontinuity of wind energy in daytime.

Current research and development efforts are required to ameliorate the efficiency and performance of PV panels as well as wind turbines, which are one of the most studied subjects of modern modular technologies.

According to the irregular character of solar and wind energies, Maximum Power Point Tracking (MPPT) algorithms are utilized for the extraction of the maximum power in order to get the optimum performance of a hybrid system. The MPPT algorithms are classified into two principal categories. One category utilizes classic methods, like hill-climbing, incremental conductance and Perturbation and Observation (P\&O). Another category uses intelligent methods, like Artificial Neural Networks (ANNs), fuzzy logic or a combination of both methods. Recently, ANNs have been used to solve very complex problems in many application fields (Elgharbi, Mezghani \& Mami, 2012). The specific solution for various non-linear and complex systems can be determined using the function and results of ANNs (Hadjab, Berrah \& Abid, 2012).

The main contribution of this article is to implement a controller, on a Field-Programmable Gate Array (FPGA), named ANN MPPT. The power management controller is used to 
command, supervise and synchronize, in real time, the power output of a system consisting of two energy sources (wind and solar).

The first objective of the present work is to develop intelligent ANN controllers for the proposed hybrid system and to control the duty cycle (d) and the proportional-integral of solar energy and wind turbine, respectively. For the wind system, in order to extract the Maximum Power Point (MPP), the boost converter is controlled to check, for every wind speed, the optimum rotor speed. In the same way, for the solar system, the PV-array MPP is variational. Indeed, the boost converter is adjusted to determine the optimum Pulse Width Modulation (PWM) in accordance with the current-voltage characteristics. The solar and wind energy systems are designed using MATLAB/SIMULINK.

The second objective of this paper is to implement, on a FPGA, the ANN MPPT controller proposed in the hybrid wind-solar conversion system using a Xilinx System Generator (XSG) a plug-in to SIMULINK which actually allows designing and simulating a system through the use of MATLAB/ SIMULINK and Xilinx library. XSG handles most routing and placement timing. Furthermore, FPGA offers many advantages compared with a sequential machine microcontroller. It achieves the current operation where the instructions are executed continuously and simultaneously. FPGA is suitable as a rapid implementation controller which can be programmed to do several types of digital functions.

To track MPP, various MPPT algorithms useful for the fixation and extraction of the maximum power exist in the specialized literature. These methods are addressed in different ways. In [11], to command the MPP of a hybrid system, Izadbakhsh et al. proposed an ANN genetic controller with three layers for various PV irradiation, as well as a fuzzy logic controller with three trapezoidal membership, a min-max method and a centroid mechanism for a wind turbine. In [19], Prakash and Sankar evaluated and improved the quality of the power generated by the hybrid system. They used an Elman neural network algorithm to track and improve MPPT. The rated power for the used wind turbine was $5 \mathrm{Kw}$ with a fixed atmospheric wind speed $12 \mathrm{~m} / \mathrm{s}$. In [1], a standalone PV-wind energy conversion system was sized and optimized. $\mathrm{P} \& \mathrm{O}$ and fuzzy logic algorithm controllers were used to get the optimal mechanical speed of the turbine and to define the Direct Current-Direct Current (DC-DC) converter duty cycle. In [21], a hybrid ANN-fuzzy logic controller was developed to adjust the root mean square error for MPPT. The optimization algorithm used was ANFIS-FLOWER pollination for brushless DC motor-driven PV pumping systems. The structure of ANN-fuzzy controller was composed of 25 fuzzy rules and five ANN layers. In [22], Po-Chen et al. proposed two methodology algorithms for MPPT. The first one would define the input membership function setting values using the solar- cells power-voltage curve. The second one would use another method based on the Particle Swarm Optimization PSO algorithm with the purpose of optimizing the input membership function. The results show that the second asymmetrical Fuzzy-logic controller which is based on MPPT algorithm, had the maximum fitness value.

In recent years, several countries are moving towards renewable energies for the protection of the environment and reducing energy costs. In the literature, research related to the control of photovoltaic-wind hybrid systems is too little compared to the importance of its systems. moreover, control techniques by artificial intelligence are often used in the field of image processing and robotics. Therefore, this study will serve as an example for the use of ANN, with several structures, as a control method. This study will also provide a new approach to the problems related to the control of agricultural pumping systems in the literature. This paper begins with an introduction which presents the contribution of the present work as well as the background of hybrid wind-solar system. The next section is dedicated to the related works. Section 3 is the global model description of hybrid system components. Section 4 describes the XSG tools used for ANN controllers and presents the conception and the hardware architecture of the different hybrid system blocks prepared for the implementation on FPGA. Section 5 details the simulation results and discussions. Section 6 presents and discusses the implementation of an ANN controller on FPGA. The conclusions are given in the last section of this paper. 


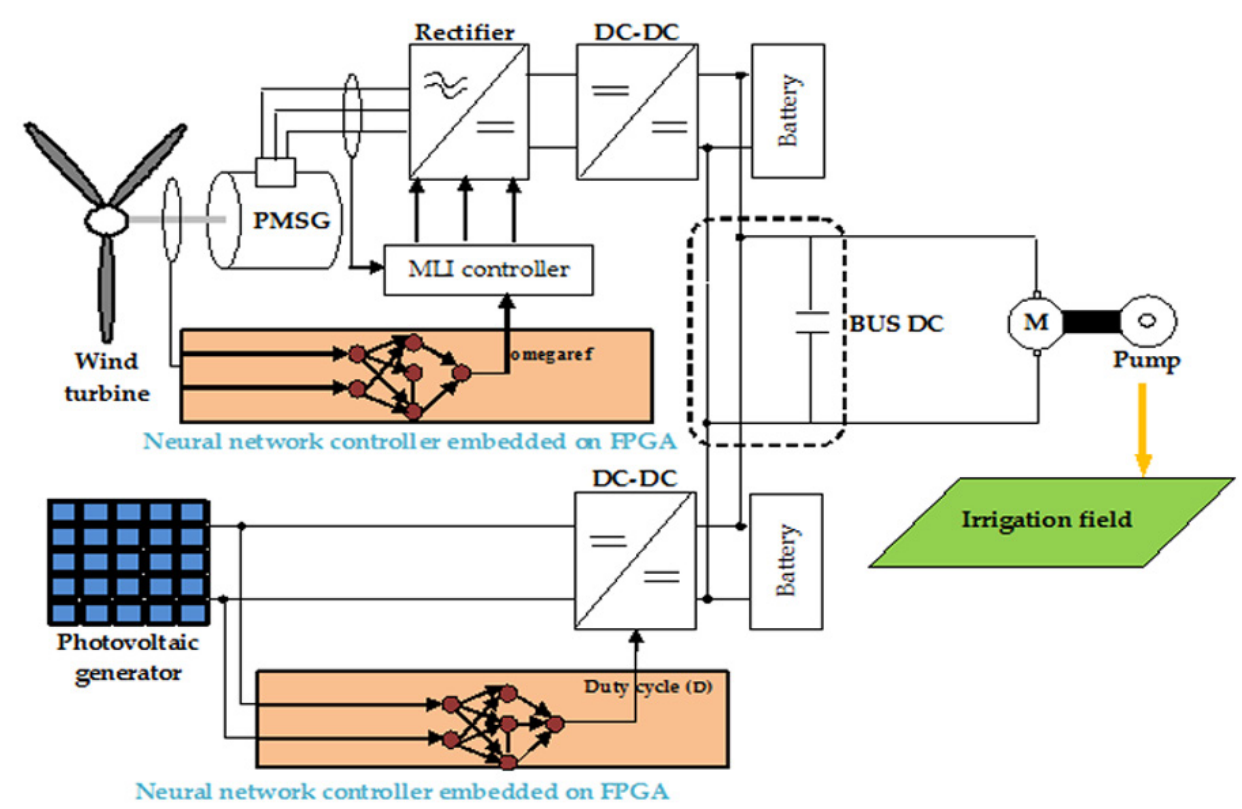

Figure 1. Global scheme of hybrid pumping system

\section{Proposed Pumping System}

The global scheme of the wind-solar system, presented in this article, is shown and detailed in Figure 1. Its principal blocks are: one Permanent Magnet Synchronous Generator (PMSG), a continuous DC-DC bus, one wind turbine, a PV generator, boost converters and ANN controllers.

For the management of the energy produced, we use a DC-DC converter for each energy source directly connected to the batteries in order to maintain the values of the voltage. In addition, we use control loops in the wind subsystem to facilitate the neural control of the optimal speed.

\subsection{Solar Energy}

In the beginning, the main elements of the equivalent circuit of one PV cell are an ideal diode, a parallel resistor $\left(\mathrm{R}_{\mathrm{P}}\right)$, a series resistor $\left(\mathrm{R}_{\mathrm{S}}\right)$ and one ideal current source (IPV). In addition to that, PV cells are characterized by their opencircuit voltage and their short-circuit current. The equation of the current voltage (IPV -VPV) is derived from the Kirchhoff's law (Bharath \& Srinivasarao, 2016). It is given by equation (1).

$$
\mathrm{I}=\mathrm{I}_{\mathrm{ph}}-\mathrm{I}_{\text {or }}\left(\frac{\mathrm{T}_{\mathrm{C}}}{\mathrm{T}_{\mathrm{ref}}}\right)^{3} e^{\frac{q E_{g}}{n K}\left(\frac{1}{T_{\text {ref }}}-\frac{1}{T_{c}}\right)}\left[e^{\frac{q\left(v+I R_{s}\right)}{n K T_{c}}-1}\right]-\frac{V+I R_{s}}{R_{p}}
$$

Figure 2 describes the scheme of the solar energy conversion system studied in this paper.

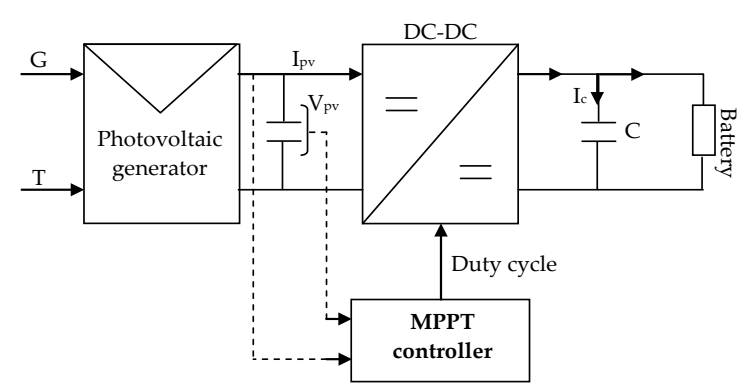

Figure 2. Solar energy conversion system

The PV module includes many series or parallel connected solar cells. For a PV module, with $N_{s}$ cells in series and $N_{p}$ cells in parallel, the I-V characteristic of a practical PV array is given by equation (2):

$$
\begin{aligned}
& \mathrm{I}_{\mathrm{pv}}=\mathrm{n}_{\mathrm{p}} \mathrm{I}_{\mathrm{ph}}-\mathrm{n}_{\mathrm{p}} \mathrm{I}_{\mathrm{or}}\left(\frac{\mathrm{T}_{\mathrm{C}}}{\mathrm{T}_{\mathrm{ref}}}\right)^{3} e^{\frac{q E_{g}}{n K}\left(\frac{1}{T_{r e f}}-\frac{1}{T_{c}}\right)}\left[e^{\frac{q\left(v+I R_{s}\right)}{n K T_{c} n_{s}}-1}\right] \\
& -\frac{V+R_{s} I}{R_{p}}
\end{aligned}
$$

Table 1 presents the electric specifications of the photovoltaic generator.

Table 1. PV generator specifications (Jemaa et al., 2018)

\begin{tabular}{|l|l|}
\hline Number of cells in parallel and series & $1 * 36$ \\
\hline Current max point & $3.25 \mathrm{~A}$ \\
\hline Voltage max point & $16.8 \mathrm{~V}$ \\
\hline Short circuit current & $3.56 \mathrm{~A}$ \\
\hline Short circuit voltage & $21.6 \mathrm{~V}$ \\
\hline Power & $60 \mathrm{~W}$ \\
\hline
\end{tabular}




\subsection{Wind Energy}

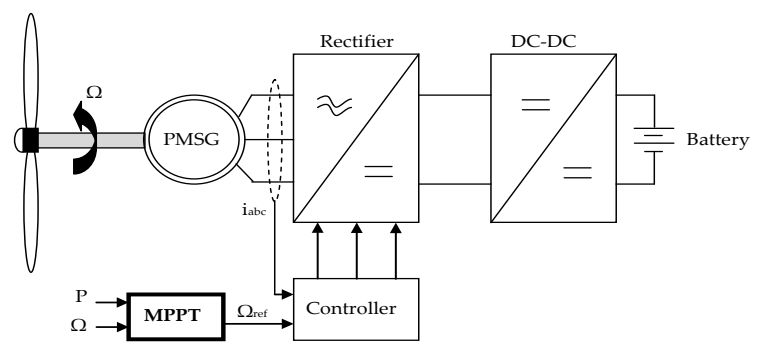

Figure 3. Schematic of wind energy system

Figure 3 describes the schematic model of the wind system studied in this work. Its principal blocks are: a battery, a BOOST converter, a rectifier, PMSG and a wind turbine. The power expression is as follows:

$\mathrm{P}=\frac{1}{2} \Pi \rho \mathrm{C}_{\mathrm{P}}(\lambda, \beta) \mathrm{R}^{2} V_{v}^{3}$

where $\mathrm{V}_{\mathrm{v}}$ is the wind speed, $\mathrm{Cp}$ is the power coefficient, $\rho$ is the air density, $\mathrm{R}$ is the radius of turbine, and $\beta$ and $\lambda$ are the pitch angle of the turbine and the tip speed ratio respectively. $\mathrm{C}_{\mathrm{p}}$ varies with $\lambda$ and it is maximal at the particular $\lambda_{\text {opt }}$. It is expressed by equation (4).

$\mathrm{C}_{\mathrm{p}}(\lambda)=-0.2121 \lambda^{3}+0.0856 \lambda^{2}+0.2539 \lambda$

Let $\beta$ be equal to zero and $\lambda$ be expressed by:

$\lambda=\frac{\Omega \mathrm{R}}{\mathrm{V}_{\mathrm{v}}}$

In this equation $\Omega$ is the angular speed of the turbine. To maximize the power extracted from the wind turbine system, $\mathrm{C}_{\mathrm{p}}$, which is the power coefficient, should be maximal for $\lambda$. As a consequence, one optimal rotor speed value is required for each wind speed value, where MPP is extracted (Soetedjo, Lomi \& Puji Mulayanto, 2011). For that reason, $C_{p}$ will depend upon the wind turbine rotor speed if the wind speed remains constant. Therefore, in order to control the power output of the turbine, the rotor speed should be controlled. The analyzed wind turbine electric specifications are given in Table 2 .

Table 2. Wind turbine specifications (Jemaa et al., 2018)

\begin{tabular}{|l|l|}
\hline Radius of turbine & $0.5 \mathrm{~m}$ \\
\hline Height of turbine & $2 \mathrm{~m}$ \\
\hline Maximum power coefficient & 0.15 \\
\hline Air density & $1.2 \mathrm{~kg} / \mathrm{m}^{3}$ \\
\hline Optimal tip speed ratio & 0.78 \\
\hline
\end{tabular}

\subsection{Mathematical Model of PMSG}

The electromagnetic and voltage torque equations within a synchronous rotational (d-q)-axis reference frame are as follows:

$v_{\mathrm{d}}=\mathrm{R}_{\mathrm{s}} i_{d}+\frac{d \phi_{d}}{\mathrm{dt}}-\omega \phi_{q}$

$v_{q}=\mathrm{R}_{\mathrm{s}} i_{q}+\frac{d \phi_{q}}{\mathrm{dt}}+\omega \phi_{d}$

$\varphi_{\mathrm{d}}=\mathrm{L}_{\mathrm{d}} i_{d}+\varphi_{f}$

$\varphi_{\mathrm{q}}=\mathrm{L}_{\mathrm{q}} i_{q}$

$C_{\text {em }}=p \mathrm{i}_{\mathrm{q}}\left\{\left(L_{d}-L_{q}\right) i_{d}+\phi_{f}\right\}$

where the (d-q)-axis voltages are represented by $\mathrm{V}_{\mathrm{d}}$ and, respectively, by $\mathrm{V}_{\mathrm{q}}$, the (d-q)-axis inductances are represented by $\mathrm{L}_{\mathrm{d}}$ and, respectively, by $\mathrm{L}_{\text {, }}$, the (d-q)-axis currents represented by id and, respectively, by $i_{a}$, the rotor electrical velocity is shown by $\omega$, the per-phase stator resistance is depicted by $\mathrm{R}_{\mathrm{s}}$, the electromagnetic torque is represented by $\mathrm{C}_{\mathrm{em}}$, the permanent magnetic flux is illustrated by $\varphi_{f}$, and the machine pair-pole number is defined by $p$. It is worth mentioning that the rotor magnets produce the flux which depends on the material used for the realization of these magnets. Table 3 provides the PMSG characteristics with two pole pairs.

Table 3. PSMG specifications (Jemaa et al., 2018)

\begin{tabular}{|c|c|}
\hline Stator phase resistance $(\Omega)$ & 0.137 \\
\hline Stator phase inductance $(\mathrm{H})$ & 0.0027 \\
\hline Inertia constant $\left(\mathrm{Kg} \cdot \mathrm{m}^{2}\right)$ & 0.1 \\
\hline Friction factor $(\mathrm{Kg} \cdot \mathrm{m} / \mathrm{rad})$ & 0.06 \\
\hline
\end{tabular}

\subsection{Boost Converter Model}

This boost converter is utilized for both PV and wind generation systems. It is a power electronic device that has the capacity of providing a voltage higher than the input voltage. Its duty cycle is controlled by a selected controller. The Boost converter equations are given by:

$$
\left\{\begin{array}{l}
i_{L}=i-c_{1} \frac{d v_{i}}{d t} \\
i_{0}=(1-d) i_{L}-c_{2} \frac{d v_{0}}{d t} \\
v_{i}=(1-d) v_{0}+L \frac{d i_{L}}{d t}
\end{array}\right.
$$

The relationship between input and output voltages is given by equation (12):

$$
\frac{V_{0}}{\mathrm{~V}_{\mathrm{i}}}=\frac{1}{(1-\mathrm{d})}
$$

where $\mathrm{V}_{\mathrm{i}}$ is the output voltage, $\mathrm{V}_{0}$ is the voltage of boost converter, and $\mathrm{d}$ is the duty cycle. 


\subsection{Pulse-width Modulation}

The input voltages of PWM are expressed by:

$$
v_{\mathrm{n}}=\mathrm{V}_{\mathrm{d}}\left(S_{n}-\frac{1}{3} \sum_{n=a}^{c} S_{n}\right)
$$

where $\mathrm{S}_{\mathrm{n}}=0$ or 1 are the states of the switches.

The equation of the DC bus current is given by:

$C \cdot \frac{d V_{\mathrm{dc}}}{\mathrm{dt}}=\mathrm{i}_{\mathrm{c}}$

with $i_{c}=i_{c h}-i_{d c}$

Thus, the expression of the coupling between alternating and continuous sides as a function of the state of the switches is given by:

$$
C \cdot \frac{d V_{\mathrm{dc}}}{\mathrm{dt}}=\mathrm{S}_{\mathrm{a}} i_{a}+\mathrm{S}_{\mathrm{b}} i_{b}+\mathrm{S}_{\mathrm{c}} i_{c}-i_{d c}
$$

\subsection{MPP Control}

The MPPT controller is the most important element in the hybrid system which allows the search of the MPP in various atmospheric conditions. Technically, its role is to converge the duty factor value for the PWM block to the power converter output. Many artificial techniques for MPPT have been developed and implemented in different circuit supports. The most popular classic methods are hill climbing, (P\&O) and incremental conductance. Fuzzy logic and ANNs are considered modern techniques. Concerning a wind subsystem, the MPPT techniques depend on the initial reference rotor speed, and for a solar subsystem they depend on the initial reference voltage. The corresponding power values of the two wind and solar systems are measured. When these values do not fit the maximal power, their initial reference values will be in fact incremented and decremented by one step. In case such an adjustment results in the growth of their output power, after that the following adjustment will be made in the same direction. The other way around is also right. The above steps are repeated until MPP of wind and solar systems is reached.

\subsection{Neural Network Controller}

To get an ANN, it is important to combine two or more artificial neurons which can be structured in several layers and connected in different ways. The network architecture is determined by the way that neurons are connected to solve complex problems in different domains. These interconnections can be done with different possible topologies divided into two classes: feedforward and feedback networks. Multi Layer Perceptron (MLP) ANNs are the most known and utilized feedforward neural networks which have only one condition: the direction of the transmitted information must be from inputs to outputs without back-loops (Nagaraj \& Panigrahi, 2015).

The architectures of a simple neural network of solar and wind systems consist of using MLP networks which include input, hidden and output layers with respected weight and transfer functions. In this case, the neuron network knows that the desired output as well as the weight coefficients are adjusted and calculated by a controller to reach the specific output. In every hidden node, the products of weights and inputs should be summed in order to be compared with a fixed threshold. If the value is above, the neuron takes an activated value. Otherwise, the neuron takes the deactivated one (Ons et al., 2015). Added to that, the algorithm utilized to train this neural network is back-propagation. It needs only inputs as well as a desired output to adapt the weight.

\section{XSG}

\subsection{Introduction}

A XILINX library from MATLAB/SIMULINK is used to generate a FPGA programming file. It is worth mentioning that the FPGA implementation steps are automatically completed. These steps are synthesis, place and route. The principal advantage of using XSG for FPGA implementation is that Xilinx components provide a close integration with MATLAB/ SIMULINK in practice. The System Generator setting window defines the FPGA board type that will be used in the present system, and provides some additional options for analysis, compilation and clock speed. In addition to that, System Generator allows a faster prototyping and designing in terms of high-level programming, through the use of more than 90 DSP building blocks. There are several components from the MATLAB/SIMULINK library that enable direct programming: C code, M-code, and VHDL. System Generator projects can be also transferred directly into the FPGA support as an executable bit stream file, and can generate a VHDL code for additional optimizations or integration with the existing projects without using Xilinx ISE. 


\subsection{Neural Network Controllers of Solar and Wind Energy using XSG}

The neural network is trained through the use of a MATLAB file (.m). The architecture of neural network (number of layers and neurons, activation function) is defined using the newff function. During the training of the MLP, the learning rate iteratively adjusts the network weights and biases to reach the desired output. After training, backpropagation learning rule defines those different weights and biases of the network. The neural network controller in MATLAB/SIMULINK can be designed using arithmetic blocks (Elgharbi, Mezghani \& Mami, 2012). Using the Xilinx library, the proposed architecture of the neural network controller can be designed on XSG (Figure 4, Figure 5).

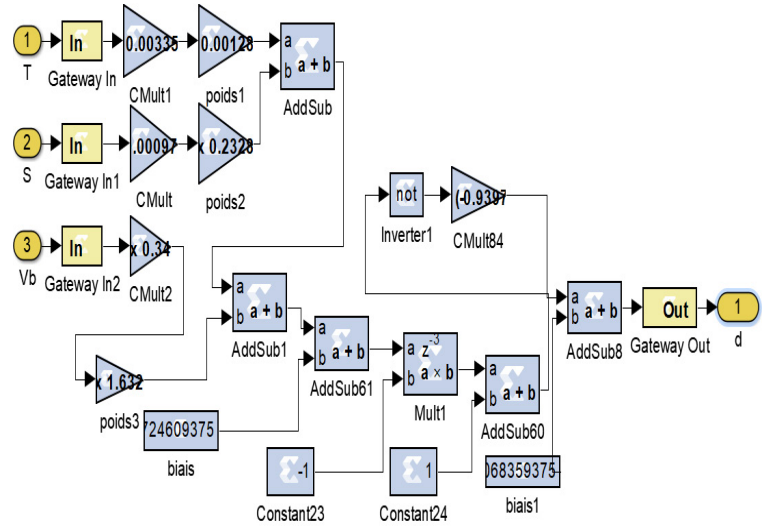

Figure 4. Neural network controller for solar system on XSG

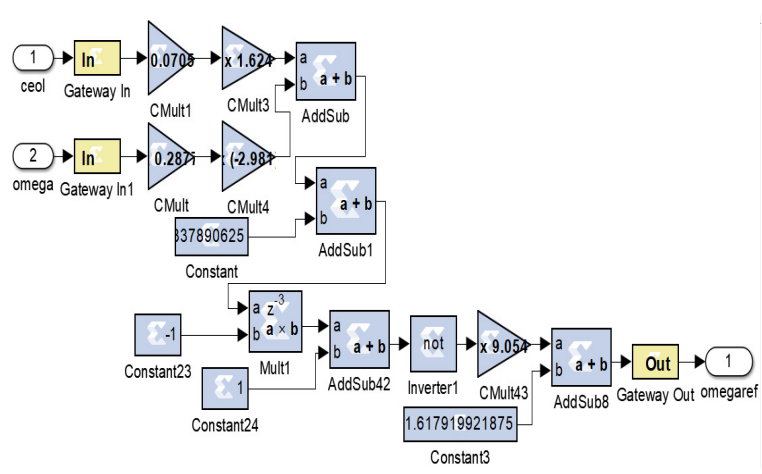

Figure 5. Neural network controller for wind system on XSG

All Xilinx blocks should be linked between two gateways: 'gateway in' and 'gateway out' where any method can be designed. All Xilinx blocks work on a fixed point. On the other hand, a real world signal works on a floating point. In this case, the gateway-in and gateway-out blocks act, in a way or another, as translators in order to convert a real world signal into a desired form.

\section{Hardware Co-simulation Results}

Figure 6 shows the designed model of the hybrid system with an ANN controller, in a MATLAB/ SIMULINK environment, To compare and analyze the results of the ANN MPPT controllers designed on MATLAB/SIMULINK and XSG, several ANNs are analyzed with various atmospheric conditions for the PV system (Table 4).

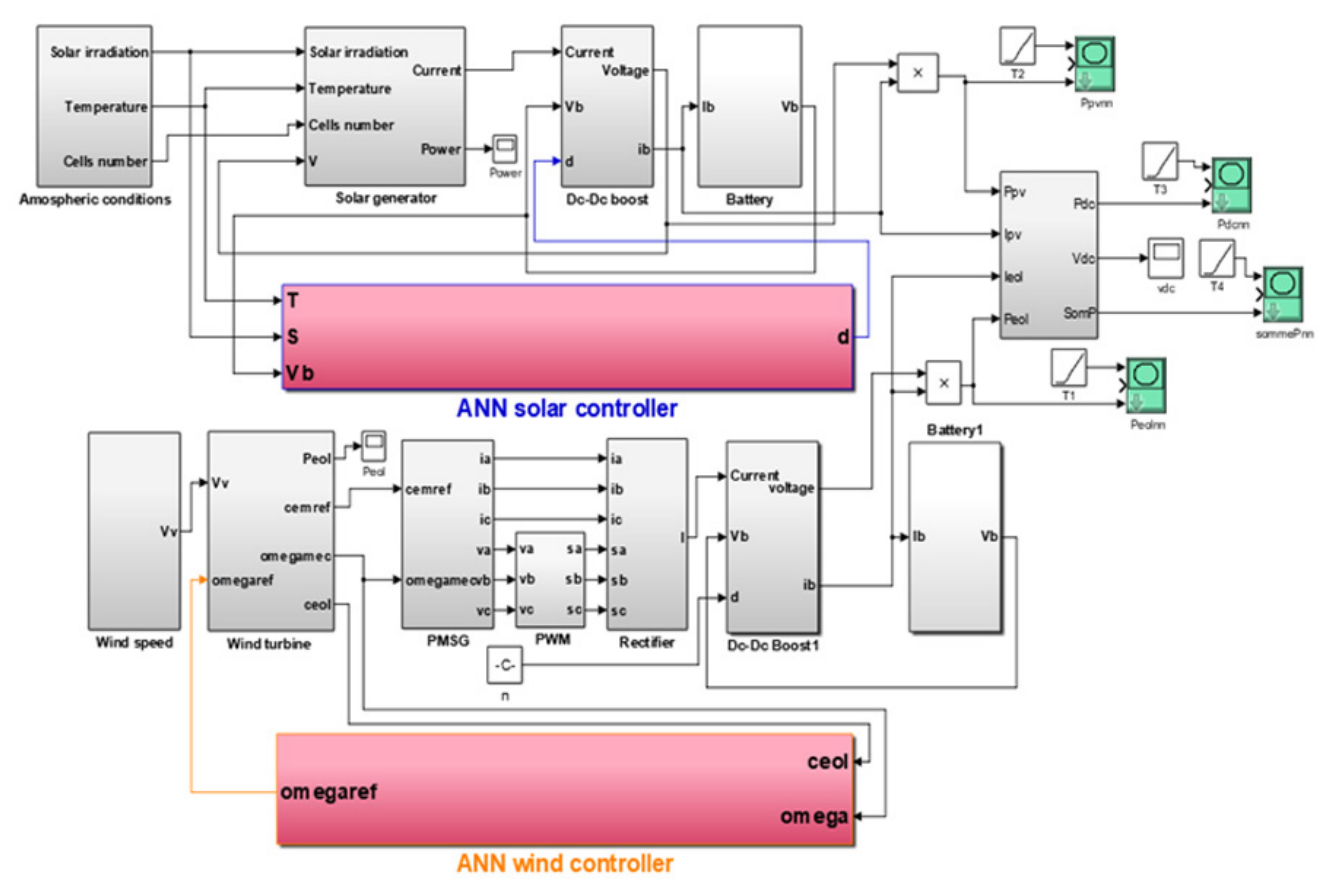

Figure 6. MATLAB/SIMULINK model of hybrid energy conversion system 
Table 4. Atmospheric conditions for PV system

\begin{tabular}{|c|c|c|}
\cline { 2 - 3 } \multicolumn{1}{c|}{} & $\mathbf{G}\left[\mathbf{W} / \mathbf{m}^{\mathbf{2}}\right]$ & $\mathbf{T}[\mathbf{K}]$ \\
\hline $\mathbf{1}$ & 600 & 298 \\
\hline $\mathbf{2}$ & 1000 & 280 \\
\hline $\mathbf{3}$ & 900 & 290 \\
\hline $\mathbf{4}$ & 750 & 285 \\
\hline $\mathbf{5}$ & 800 & 288 \\
\hline $\mathbf{6}$ & 700 & 292 \\
\hline $\mathbf{7}$ & 900 & 300 \\
\hline $\mathbf{8}$ & 850 & 295 \\
\hline $\mathbf{9}$ & 980 & 278 \\
\hline $\mathbf{1 0}$ & 700 & 295 \\
\hline
\end{tabular}

The expression of the variable speed wind is expressed in equation (16)

$$
\begin{aligned}
& V_{v}(t)=7.5+0.2 \sin (0.1047 t)+2 \sin (0.2665 t) \\
& +\sin (1.2930 t)+0.2 \sin (3.6645 t)
\end{aligned}
$$

The ANN structures proposed in this paper are given in Table 5.

For both wind and solar systems, the first ANN structure includes just one neuron and one sigmoid activation function in the first hidden layer, as well as one single neuron and one linear activation function in the second hidden layer. In the second analyzed ANN structure, the number of neurons of the first layers is increased and linear activation functions are used in the second layers for both wind and solar systems. The third ANN structure contains three hidden layers and one neuron for both wind and solar systems.

In addition, sigmoid activation functions are used in both first layers, and linear activation functions are used in the third one for both wind and solar systems. The architecture in the fourth and fifth analyzed ANN structures is the same as the one used in the third one and the number of neurons of the first layers is raised. All the ANN controller structures are modeled using MATLAB/ SIMULINK. The obtained power curves of the simulated hybrid system are shown in Figure 8.

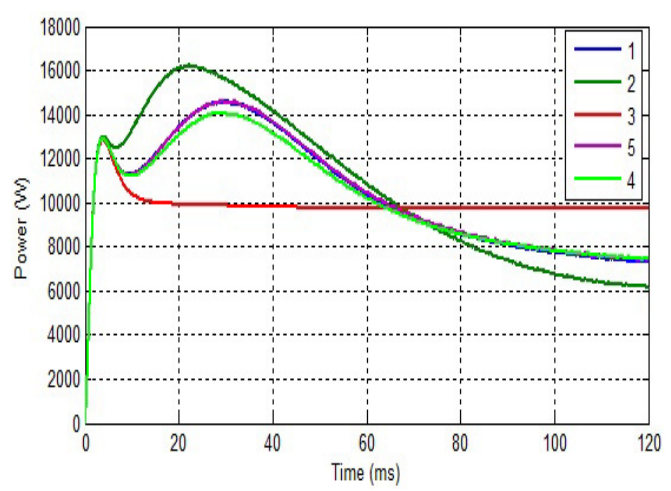

Figure 7. Power curves obtained with five neuronal structures.

These ANN structures give good power values that can satisfy the continuous supply of the hybrid system (Figure 7).

The five curves reach their MPP at different values of time. The most quickly ones are considered. The curves of different structures show that the second structure reaches its MPP value first. This structure will be used on the hybrid system designed with XSG.

The hybrid system designed with XSG blocks is simulated with the goal of comparing the results obtained by means of SIMULINK with those achieved using a designed hardware module.

Table 5. Structures of analyzed ANN controllers

\begin{tabular}{|c|c|c|c|c|c|c|c|}
\cline { 2 - 8 } \multicolumn{1}{c|}{} & \multirow{2}{*}{$\begin{array}{c}\text { System } \\
\text { type }\end{array}$} & $\begin{array}{c}\text { Number of } \\
\text { neurons }\end{array}$ & $\begin{array}{c}\text { Activation } \\
\text { function }\end{array}$ & $\begin{array}{c}\text { Number of } \\
\text { neurons }\end{array}$ & $\begin{array}{c}\text { Activation } \\
\text { function }\end{array}$ & $\begin{array}{c}\text { Number of } \\
\text { neurons }\end{array}$ & $\begin{array}{c}\text { Activation } \\
\text { function }\end{array}$ \\
\cline { 2 - 8 } & Wind & 1 & Sigmoidal & 1 & Linear & - & \\
\cline { 2 - 8 } & Solar & 1 & Sigmoidal & 1 & Linear & - & \\
\hline \multirow{2}{*}{$\mathbf{2}$} & Wind & 20 & Sigmoidal & 1 & Linear & - & \\
\cline { 2 - 8 } & Solar & 20 & Sigmoidal & 1 & Linear & - & Linear \\
\hline \multirow{2}{*}{$\mathbf{3}$} & Wind & 1 & Sigmoidal & 1 & Sigmoidal & 1 & Linear \\
\cline { 2 - 8 } & Solar & 1 & Sigmoidal & 1 & Sigmoidal & 1 & Linear \\
\hline \multirow{2}{*}{$\mathbf{4}$} & Wind & 30 & Sigmoidal & 1 & Sigmoidal & 1 & Linear \\
\cline { 2 - 8 } & Solar & 30 & Sigmoidal & 1 & Sigmoidal & 1 & Linear \\
\hline \multirow{2}{*}{$\mathbf{5}$} & Wind & 10 & Sigmoidal & 1 & Sigmoidal & 1 & Linear \\
\cline { 2 - 8 } & Solar & 10 & Sigmoidal & 1 & Sigmoidal & 1 & layer \\
\hline
\end{tabular}


Table 6. Performance comparison

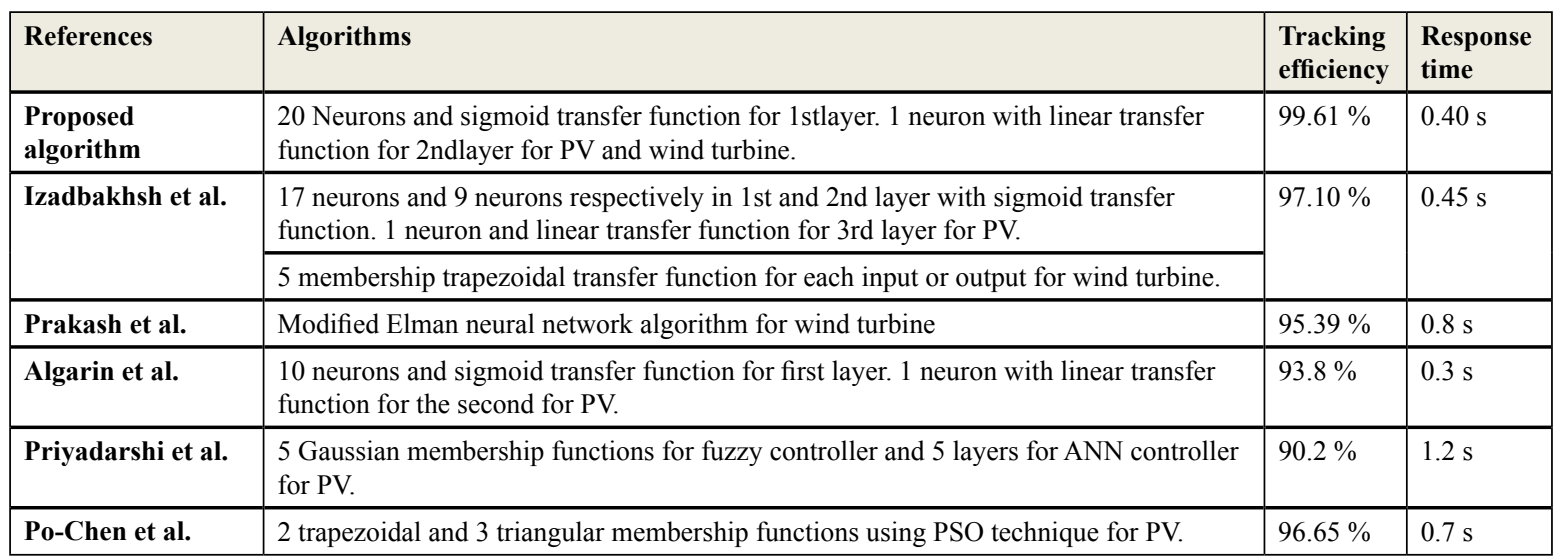

Figure 8 shows the power curves obtained with the proposed ANN controller with the one designed on XSG. The system stabilization with XSG is quicker due to parallel processing.

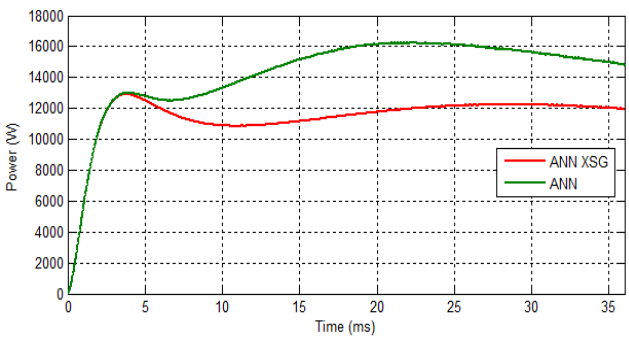

Figure 8. Power curves obtained with ANN controller in Simulink and with the one designed on XSG

A comparison between other studies and the high efficiency of the proposed model is required to be made. Examples of some FPGA implementations of ANN and fuzzy logic algorithms as well as their main characteristics are presented in Table 6 .

The references chosen for comparison use different topologies. The values of tracking the efficiency and response time of references compared with the proposed algorithm satisfy and validate the model designed in this paper. After verifying the progress of the system, the implementation of the controllers on FPGA circuits represents the next phase.

\section{FPGA Implementation}

In this work, FPGAs are a good choice because of their characteristics such as high performance and re-configurability and low energy dissipation (Elamaran \& Rajkumar, 2012). In order to implement the design of the hybrid energy conversion system in an FPGA board, the entire module is required to be converted to a FPGA synthesizable module. At first, at the system level, using floating- point Simulink blocksets, an algorithm is modeled and simulated. A design necessary to implement the system on FPGA is presented with XSG blocks. By clicking on the system generator token, a window appears. After opening a token dialog box of System Generator, the compilation type should be selected. The second step is to define the FPGA part (Virtex6XC6VSX315T). The characteristics of the used FPGA are presented in Table 7.

Table 7. Characteristics of FPGA Virtex-6

\begin{tabular}{|c|c|c|}
\hline $\begin{array}{l}\text { Total number of } \\
\text { configuration bits }\end{array}$ & \multicolumn{2}{|c|}{$104,465,888$} \\
\hline \multirow{2}{*}{$\begin{array}{c}\text { Configurable logic } \\
\text { blocks }\end{array}$} & Slices & 49.200 \\
\hline & Max RAM & $5.090(\mathrm{~Kb})$ \\
\hline Total I/O banks & \multicolumn{2}{|c|}{18} \\
\hline $\begin{array}{c}\text { Interface blocks for } \\
\text { PCI Express }\end{array}$ & \multicolumn{2}{|c|}{2} \\
\hline Max user I/O & \multicolumn{2}{|c|}{720} \\
\hline Logic cells & \multicolumn{2}{|c|}{314.880} \\
\hline
\end{tabular}

After that, the tool and the HDL language utilized for synthesizing the design and for compilation (VHDL) are specified. The System Generator creates a HDL test bench. Added to that, the design is synthesized and implemented.

At this time, the period (ns) of the system clock and the hardware clock pin location can be identified. Using the token dialog box of the System Generator, the code generator is called when clicking on the 'generate' button.

During the Register Transfer Level (RTL) step, the synthesizer observes the syntax and programming errors. Next, it transforms the VHDL program 
into basic electronic elements like logic gates and flip-flops. It also permits imposing technological constraints (User constraints): for example to set the speed of operation (Create timing Constraints), to delimit the zone of the FPGA circuit in which the routing must be done (Create Area constraints) or to assign the pins inputs / outputs (Assign Package Pins). The View RTL Schematic tool allows viewing the equivalent patterns generated by the synthesizer for each block of the program as well as the relationship between different blocks in the main program. 'Device utilization summary', presented in Table 8 , depicts the information that is in close relation with the device utilization analysis. The total power dissipation is $4,605 \mathrm{~W}$ and the maximum frequency is $62.8 \mathrm{~Hz}$.

Table 8. Device utilization summary

\begin{tabular}{|l|c|c|}
\hline \multirow{2}{*}{\multicolumn{1}{|c|}{ Logic use }} & \multicolumn{2}{c|}{ ANN controller } \\
\cline { 2 - 3 } & Utilization & Used \\
\hline Number of STARTUPs & 1 & $100 \%$ \\
\hline Number of DSP48E1s & 51 & $3 \%$ \\
\hline Number of bonded IOBs & 425 & $70 \%$ \\
\hline Number of occupied slices & 835 & $1 \%$ \\
\hline Number used as memory & 116 & $1 \%$ \\
\hline Number of slice LUTs & 2,782 & $1 \%$ \\
\hline Number of slice registers & 457 & $1 \%$ \\
\hline
\end{tabular}

\section{Conclusion}

In this paper, one hybrid wind-solar energy system based on ANN controllers has been presented

\section{REFERENCES}

1. Algarín, C. R., Hernández, D. S. \& Lea, D. R. (2018). A low-cost Maximum Power Point Tracking System Based on Neural Network Inverse Model Controller, Electronics, 7(4), 17.

2. Argaw, N. (2004). Renewable Energy Water Pumping Systems Handbook, National Renewable Energy Laboratory, Subcontractor Report, p 128.

3. Avedian-Gonzalez, G., Gonzalez-Potes, A., Ibarra-Junquera, V., Mata-Lopez, W. A. \& Escobar-del Pozo, C. (2018). Smart Control System to Optimize Time of Use in a SolarAssisted Air-Conditioning by Ejector for Residential Sector, Applied Sciences, 8(3), 350. and implemented on FPGA. The MATLAB/ SIMULINK system design is used to model the different blocks of the system with five ANN controller structures. The chosen structure is composed of two hidden layers. The first layer contains 20 neurons with a sigmoid transfer function. The second one contains one neuron with a linear transfer function. Then the two solar and wind controllers with XSG are designed. The XSG simplifies the complicated programming and makes it easy for the designers to accelerate simulation and at the same time to test the design in hardware. In fact, due to the advantage of the parallel XSG structure, the stabilization of the system is quicker. The MPP achieving time and tracking efficiency are about $4 \mathrm{~ms}$ and $99.61 \%$ respectively. The experimental simulation results show good performances in comparison with other existing systems in terms of the used strategy as a function of achieved tracking time and efficiency.

At the end of this article, the implementation on FPGA Virtex-6-XC6VLX315T hardware of the proposed system was accomplished by using the Xilinx ISE design. Finally, the utilization of MATLAB/SIMULINNK and XSG simplifies the hardware implementation. In perspective, this work can be extended by utilizing this system in the power supply of a complete farm.

4. Belaji Damodhar, T. S. \& Senthil Kumar, A. (2016). Implementation of FPGA Based Hybrid Power Generator for PV and Win Grid Applications, Circuits and Systems, 7, 4280-4290.

5. Bharath, N. \& Srinivasarao, R. (2016). Advanced Hybrid Wind-Solar Power System with Maximum Power Point Tracking Algorithms, Imperial Journal of Interdisciplinary Research, 2(10), 1100-1113.

6. Elamaran, V. \& Rajkumar, G. (2012). FPGA implementation of point processes using Xilinx System Generator, Journal of Theoretical and Applied Information Technology, 41(2), 201-206. 
7. Elgharbi, A., Mezghani, D. \& Mami, A. (2012). A Maximum Power Point Tracking Method Based on Artificial Neural Network for PV System, International Journal of Advances in Engineering \& Technology, 5(1), 130-140.

8. Gupta, A., Vaishnav, H. \& Garg, H. (2015). Image Processing Using Xilinx System Generator (XSG) in FPGA, International Journal of Research and Scientific Innovation, 2(9), 119-125.

9. Hadjab, M., Berrah, S. \& Abid, H. (2012). Neural network for modeling solar panel, International Journal of Energy, 6(1), 9-16.

10. Hui, J., Bakhshai, A. \& Jain, P. K. (2010). A Hybrid Wind-Solar Energy System: A New Rectifier Stage Topology. In IEEE Applied Power Electronics Conference, Feb. 21-25, 2010, Palm Springs, CA (pp.155-161).

11. Izadbakhsh, M., Rezvan, A. \& Gandomkar, M. (2015). Dynamic response improvement of hybrid system by implementing ANN-GA for fast variation of photovoltaic irradiation and FLC for wind turbine, Electrical Engineering, 64(2), 291-314.

12. Jemaa, A., Zarrad, O., Craciunescu, A. \& Popescu, M. (2016). Comparison of fuzzy and neuro-fuzzy controllers for maximum power point tracking of photovoltaic modules, Renewable Energy and Power Quality Journal, 1(14), 796-800.

13. Jemaa, A., Zarrad, O., Hajjaji, M. A. \& Mansouri, M. N. (2018). Hardware Implementation of a Fuzzy Logic Controller for a Hybrid Wind-Solar System in an Isolated Site, International Journal of Photoenergy, p 21.

14. Lalouni, S., Rekioua, D., Idjdarene, K. \& Tounzi, A. M. (2014). Comparative Study Between MPPT Algorithms Applied to Wind Energy Conversion System, IPCO, 2.

15. Marisarla, C. \& Ravi Kumar, K. (2013). A Hybrid Wind and Solar Energy System with Battery Energy Storage for an Isolated System, International Journal of Engineering and Innovative Technology, 3(3), 99-104.

16. Nagaraj, R. \& Panigrahi, B. K. (2015). Simulation and Hardware Implementation of FPGA Based Controller for Hybrid Power System, International Journal of Electrical Energy, 3(2), 86-93.
17. Ons, Z., Aymen, J., Craciunescu, A. \& Popescu, M. (2015). Comparison of HillClimbing and Artificial Neural Network MPPT Techniques for Photovoltaic Modules. In Proceedings of the 2015 Second International Conference on Mathematics and Computers in Sciences and in Industry (MCSI) (pp.19-23).

18. Pavankumar Reddy, K. \& Venu Gopala Rao, M. (2015). Modeling and Simulation of Hybrid Wind Solar Energy System using MPPT, Indian Journal of Science and Technology, 8(23).

19. Prakash, N. \& Ravikumar, R. (2012). A stand Alone Hybrid Power Generation System by MPPT Control Based on Neural Networks, International Journal of Science and Research, 1(3), 108-116.

20. Prakash, N. \& Sankar, K. (2012). Neural Network-Control Scheme for Grid Connected Hybrid Power Generation System for Power Quality Improvement, IOSR Journal EEE, 3(1), 53-59.

21. Priyadarshi, N., Padmanaban, S., MihetPopa, L., Blaabjerg, F. \& Azam, F. (2018). Maximum Power Point Tracking for Brushless DC Motor-Driven Photovoltaic Pumping Systems Using a Hybrid ANFISFLOWER Pollination Optimization Algorithm, Energies, 11, 1067-1083.

22. Po-Chen, C., Bo-Rei, P., Yi-Hua, L., Yu-Shan, C. \& Jia-Wei, H. (2015). Optimization of a Fuzzy-Logic-Control-Based MPPT Algorithm Using the Particle Swarm Optimization Technique, Energies, 8, 5338-5360.

23. Saadi, A. \& Moussi, A. (2003). Neural Network Use in the MPPT of Photovoltaic Pumping System. In ICPWE (pp. 39-45).

24. Sharma, S. \& Pritamdas, K. (2016). FPGA Based Efficient Median Filter Implementation Using Xilinx System Generator, International Journal of Innovation Research in Science Engineering and Technology, 5(5), 8946-8952.

25. Soetedjo, A., Lomi, A. \& Puji Mulayanto, W. (2011). Modeling of Wind Energy System with MPPT Control. In International Conference on Electrical Engineering and Informatics. 